\title{
Proposal for Plasma Wave Oscillator
}

\author{
Ashutosh Sharma ${ }^{1,2,3^{*}}$ \\ ${ }^{1}$ Department of Education, University of Lucknow, Lucknow, India; ${ }^{2}$ Centre for Plasma Physics School of Mathematics and Physics, \\ Queen's University, Belfast, UK; ${ }^{3}$ Institute of Photonics Technologies, National Tsing Hua University, Hsinchu, Taiwan. \\ Email: *a_physics2001@yahoo.com
}

Received August $6^{\text {th }}, 2012$; revised September $10^{\text {th }}, 2012$; accepted September $20^{\text {th }}, 2012$

\begin{abstract}
Generation of radiation by laser pulses in uniform plasma is generally minimal. However, if one considers propagation in corrugated plasma channels, the condition for radiation generation can be met due to the inhomogeneity of the plasma channel and the presence of guided waves with subluminal phase velocities. For establishing a large amplitude plasma wave driven by moderate-power laser, one has to implement a distributed-feedback structure into the plasma (Plasma Wave Oscillator) with the feedback matching the plasma resonance. In this note the theoretical analysis for plasma waves driven by moderate-power laser for corrugated waveguide filled with pre-ionized hydrogen plasma has been developed. The growth of amplitude of plasma waves in corrugated structure, coupled to the laser and sideband fields has been investigated. The four coupled equations corresponding to laser field, sideband field and forward and backward plasma waves can be numerically solved for various parameters of the laser field, plasma density, and corrugated structure to arrive at experimental design of the Plasma Wave Oscillator, which may be used for the generation of radiation and particle acceleration.
\end{abstract}

Keywords: Laser-Plasma Interaction; Generation of Electromagnetic Radiation

\section{Introduction}

In recent years, the use of plasmas in high power microwave (HPM) devices has been actively researched. Some HPM devices are found to have a better performance when filled with plasma; as an example the backward wave oscillator (BWO) [1,2], has proved to be an efficient HPM source. A corrugated waveguide may be used to slow down the phase velocity of electromagnetic waves; in some experiments microwave radiation has been generated with considerably enhanced efficiency [3]. When a periodic structure such as a corrugated waveguide is introduced, the dispersion characteristics are drastically modified on account of the periodicity of the structure [4]. The periodic variation of the radius of waveguide may impose a periodic variation in the plasma density [5]. It is known that for a straight waveguide or channel the matched laser spot size and hence the irradiance depends strongly on the channel radius. Hence one expects that a periodic variation of the channel radius can modulate the plasma density and laser irradiance along the axis of a channel. The modulation of plasma density exerts a periodic perturbation on the dielectric constant. This density variation and hence dielectric modulation induces distributed feedback (DFB) [6,7],

${ }^{*}$ Corresponding author. which in turn influences the wave propagation and the plasma waves, driven by the laser beam.

In a recent investigation [8] a widely tunable dual mode laser diode with a single cavity structure is demonstrated. This novel device consists of a distributed feedback (DFB) laser diode and distributed Bragg reflector (DBR). Continuous wave $\mathrm{THz}$ radiation is successfully generated with low-temperature grown InGaAs photomixers from $0.48 \mathrm{GHz}$ to $1.5 \mathrm{THz}$.

The realization of corrugated plasma channel [9] allows for the guiding of laser pulses with subluminal spatial harmonics. These spatial harmonics can be phase matched to high energy electrons, making the corrugated plasma channel ideal for the acceleration of electrons. The simulations [10] performed in a corrugated preformed plasma channel obtain the accelerating gradients of several hundred $\mathrm{MeV} / \mathrm{cm}$ for laser powers much lower than required by standard laser wake field schemes. The development of corrugated slow-wave plasma guiding structures with application to quasiphase-matched direct laser acceleration of charged particles is reported by York et al. [11]. These structures support guided propagation at intensities up to $2 \times 10^{17} \mathrm{~W} / \mathrm{cm}^{2}$, limited at present by our current laser energy and side leakage. These structures remove the limitations of diffraction, phase matching, and material damage thresholds and promise 
to allow high-field acceleration of electrons over many centimeters using relatively small femtosecond lasers. It is investigated in this work by simulations that a laser pulse power of 1.9 TW should allow an acceleration gradient larger than $80 \mathrm{MV} / \mathrm{cm}$ and modest power of only $30 \mathrm{GW}$ would still allow acceleration gradients in excess of $10 \mathrm{MV} / \mathrm{cm}$.

In the present analysis a moderate-power laser (Ti: Sapphire) propagating through a corrugated waveguide (Sapphire) filled with pre-ionized hydrogen plasma has been considered. The corrugated waveguide is shown in Figure 1; the wall radius $y(z)$ of waveguide varies sinusoidally according to function,

$$
y(z)=\frac{y_{0}}{2}+\frac{h}{2} \exp \left(-j \frac{2 \pi z}{\Lambda}\right)
$$

where $h$ and $\Lambda$ are amplitude and the period of corrugation and $y_{0}$ is the average radius of corrugated structure.

The basic mechanism, considered in this study is the parametric instability [12] resulting from the resonant interaction between three non-linearly coupled waves. A laser beam, i.e. a transverse electromagnetic wave, propagating through an unmagnetized, pre-ionized underdense hydrogen plasma (frequency $\omega_{l}$ of incident light $>$ plasma frequency $\left.\omega_{p}\right)$. This incident laser beam will reflect off any electron density fluctuation, in particular perturbations related to electron plasma waves. Under condition of appropriate phase matching, the scattered and incident laser field may beat together in such a way as to reinforce the plasma wave. This reinforced plasma wave will in turn lead to a higher level of scattering, and this increased scattered light will lead to a stronger beating with the incident light, which will further amplify the plasma wave.

A periodic variation of the channel radius modulates the plasma density and laser irradiance along the axis of the channel. The modulation of plasma density exerts a

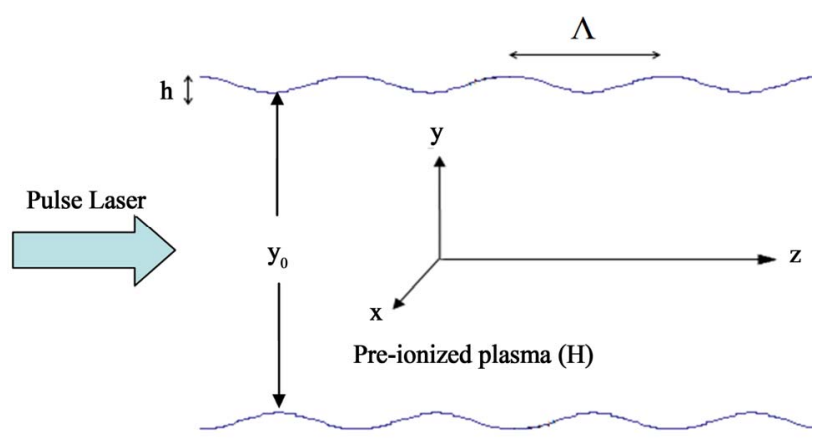

Figure 1. Plasma Wave Oscillator-where $\Lambda$ is the period of sinusoidal perturbation of radius of plasma channel generated inside the Sapphire grating structure, $h$ is the groove depth. periodic perturbation on the dielectric constant. This density variation and hence the dielectric modulation induces distributed feedback (DFB), which in turn influences the wave propagation and the plasma waves driven by laser beam.

\section{Analysis}

The electric fields of the laser are given as,

$$
\boldsymbol{E}_{l, s}(z, t)=\operatorname{Re}\left[E_{l, s}(z) \exp \left(-j \omega_{l, s} t\right)\right]
$$

and the complex amplitude of the laser and sideband field

$$
E_{l, s}(z)=A_{l, s}(z) \exp \left(j k_{l, s} z\right)
$$

where $\boldsymbol{A}_{(z)}$ is the slowly varying field envelope, subscripts 1 and s stand for laser and sideband field.

The electromagnetic wave propagation in plasma as governed by the wave equation,

$$
\nabla^{2} \boldsymbol{E}+\frac{1}{c^{2}} \frac{\partial^{2} \boldsymbol{E}}{\partial t^{2}}=-\frac{4 \pi}{c^{2}} \frac{\partial \boldsymbol{J}}{\partial t}
$$

where $\boldsymbol{J}=N_{e} \mathrm{e} \cdot \boldsymbol{v}$ is the current density.

Taking into account the laser and sideband field the plasma wave equation may be obtained as,

$$
\left[\frac{\partial^{2}}{\partial t^{2}}-v_{t h}^{2} \nabla^{2}+\omega_{p}^{2}\right] N_{e}=\nabla \cdot F_{\text {pond }}
$$

where

$$
F_{\text {pond. }}=\frac{N_{0} \cdot \mathrm{e}(\boldsymbol{v} \times B)}{m c}
$$

$\omega_{p}=\left(\frac{4 \pi N_{0} \mathrm{e}^{2}}{m}\right)^{1 / 2}$ is the plasma frequency,

$N_{0}$ is the background unperturbed plasma density, $m$ is the electronic mass, e is the electronic charge, $c$ is the speed of light in vacuum.

Following the phase matching condition $\left[\omega_{p}=\omega_{l}-\omega_{s}\right.$ and $\left.k_{p}=k_{l}-k_{s}\right]$, the coupled wave equations for laser field, sideband field and the plasma wave can be written as,

$$
\begin{aligned}
& \frac{\partial^{2} E_{l}}{\partial z^{2}}+\frac{\omega_{l}^{2}}{c^{2}} \varepsilon_{l} E_{l}=-\frac{4 \pi \mathrm{e}^{2}}{m_{e} c^{2}} \frac{\omega_{1}}{\omega_{s}} N_{e} E_{s} \\
& \frac{\partial^{2} E_{s}}{\partial z^{2}}+\frac{\omega_{s}^{2}}{c^{2}} \varepsilon_{s} E_{s}=\frac{4 \pi \mathrm{e}^{2}}{m_{e} c^{2}} \frac{\omega_{s}}{\omega_{l}} N_{e}^{*} E_{l}
\end{aligned}
$$

and

$$
\begin{aligned}
& {\left[\frac{\partial^{2}}{\partial t^{2}}-v_{t h}^{2} \nabla^{2}+\omega_{p}^{2}\right] N_{e} } \\
= & \frac{N_{0} \mathrm{e}^{2}\left(k_{s}-k_{l}\right)^{2}}{m_{e}^{2} \omega_{l} \omega_{s}} A_{l}(z) A_{s}^{*}(z) \exp \left(-j k_{p} z+j \omega_{p} t\right)
\end{aligned}
$$


The solution for the plasma wave Equation (6) can be written as,

$$
\begin{aligned}
& N_{e}=N_{e}(t, y, z)=N_{e}(y, z) \cdot \exp \left(j \omega_{e} t\right) \\
& N_{e}(y, z)=[U(y, z) \cdot F(z)]
\end{aligned}
$$

where,

$$
\begin{gathered}
U(y, z)=\operatorname{Sin}(\pi y / y(z)) \\
F(z)=A(z) \exp \left(-j \beta_{0} z\right)+B(z) \exp \left(j \beta_{0} z\right)
\end{gathered}
$$

The radius of the plasma channel can be expressed as

$$
y(z)=\frac{y_{0}}{2}+\frac{h}{2} \exp \left(-j k_{g} z\right)
$$

where $k_{g}=2 \pi / \Lambda$,

$\Lambda$ is the period of sinusoidal perturbation of radius of plasma channel generated inside the Sapphire grating structure, $h$ is the groove depth.

One can expand $y(z)$ by assuming that the perturbation is small, as follows:

$$
\frac{1}{[y(z)]^{2}}=\frac{1}{y_{0}^{2}}\left[1-\frac{2 h}{y_{0}} \exp \left(-j k_{g} z\right)\right]
$$

Using the expansion of $y(z)$ from Equation (8), one can express $U(y, z)$ as

$$
\begin{aligned}
U(y, z)= & {\left[\operatorname{Sin}\left(\frac{\pi y}{y_{0} / 2}\right)-\operatorname{Cos}\left(\frac{\pi y}{y_{0} / 2}\right)\right.} \\
& \left.\times\left(\frac{4 \pi y}{y_{0}^{2}}\right) h \exp \left(-j k_{g} z\right)\right]
\end{aligned}
$$

On substituting Equation (7a) in Equation (6) one obtains,

$$
\begin{aligned}
& {\left[\nabla^{2}+\beta^{2}\right] N_{e}(y, z) } \\
= & C_{0} \exp \left(-j k_{p} z+j\left(\omega_{p}-\omega_{e}\right) t\right)
\end{aligned}
$$

where

$$
C_{0}=\frac{N_{0} \mathrm{e}^{2}\left(k_{s}-k_{l}\right)^{2}}{m_{e}^{2} \omega_{l} \omega_{s}} A_{l}(z) A_{s}^{*}(z),
$$

and

$$
\beta^{2}=\frac{\omega_{e}^{2}-\omega_{p}^{2}}{v_{t h}^{2}} .
$$

Substituting for $N_{e}(y, z), U(y, z)$ and $F(z)$ from Equations (7a), (7c) and (9) in Equation (10), multiplying both sides of the resulting equation by $\operatorname{Sin}\left(2 \pi y / y_{0}\right)$ and integrating the equation. from $-y_{0} / 2$ to $y_{0} / 2$ and following the Bragg condition $\left[2 \beta_{0}=k_{g}\right]$, one finds two coupled equations for forward and backward plasma waves, driven be the laser and sideband field,

$$
\begin{aligned}
\frac{\partial A(z)}{\partial z}= & {\left[\frac{2 j C_{0}}{\beta_{0} \pi} \exp (-j \Delta k z)-3 \frac{\partial B(z)}{\partial z}\left(\frac{h}{y_{0}}\right)\right.} \\
& \left.-2 j B(z)\left(\frac{3 \pi^{2} h}{\beta_{0} y_{0}^{3}}+\frac{h}{4 y_{0}}\left(\frac{\beta^{2}-\beta_{0}^{2}}{\beta_{0}}\right)\right)\right]
\end{aligned}
$$

and

$$
\begin{aligned}
\frac{\partial B(z)}{\partial z}= & {\left[-\frac{2 j C_{0}}{\beta_{0} \pi} \exp (-j \Delta k z)+\frac{\partial A(z)}{\partial z}\left(\frac{h}{y_{0}}\right)\right.} \\
& \left.-2 j A(z)\left(\frac{3 \pi^{2} h}{\beta_{0} y_{0}^{3}}-\frac{h}{4 y_{0}}\left(\frac{9 \beta_{0}^{2}-\beta^{2}}{\beta_{0}}\right)\right)\right]
\end{aligned}
$$

where $\Delta k=k_{l}-k_{s}-\beta$.

On substituting the laser and sideband fields from Equation (1b) in Equations (4) and (5), one obtains the laser and sideband field coupled with the plasma wave

$$
\frac{\partial A_{s}(z)}{\partial z}=\left[-j C_{s} A(z) A_{l}(z) \exp (j \Delta k z)\right]_{0}
$$

and

$$
\frac{\partial A_{l}(z)}{\partial z}=\left[j C_{l} B(z) A_{s}(z) \exp (j \Delta k z)\right]
$$

where

$$
C_{s}=\frac{\pi^{2} \mathrm{e}^{2}}{2 m c^{2}} \frac{\omega_{s}}{\omega_{1}} \frac{1}{k_{s}}\left(1+\frac{h}{y_{0}} \exp \left(-j k_{g} z\right)\right)
$$

and

$$
C_{l}=\frac{\pi^{2} \mathrm{e}^{2}}{2 m c^{2}} \frac{\omega_{l}}{\omega_{s}} \frac{1}{k_{l}}\left(1+\frac{h}{y_{0}} \exp \left(-j k_{g} z\right)\right) .
$$

We have developed an analytic model for laser pulse propagation in a corrugated plasma waveguide, using the slowly varying envelope approximation. The background plasma was assumed to be a cold non-relativistic fluid that responded linearly to the laser field. The small enough amplitude of corrugation (as shown by $C_{s}$ and $C_{l}$ above) may impose the periodic variation on plasma wave derived by coupled laser fields. Equations (11) and (14) may be used to study the growth of plasma wave in a corrugated plasma channel and hence to design a plasma wave oscillator.

\section{Conclusion}

An analysis of the growth of amplitude of plasma waves in a corrugated structure, coupled to the laser and sideband fields has been made; the resulting equations viz. Equations (11)-(14) can be numerically solved for varying parameters of the laser field, plasma density, and corrugated structure to arrive at a design of Plasma Wave 
Oscillator which may be useful for generation of radiation and particle acceleration.

\section{REFERENCES}

[1] M. Botton and A. Ron, "Efficiency Enhancement of a Plasma-Filled Backward-Wave Oscillator by Self-Induced Distributed Feedback," Physical Review Letters, Vol. 66, No. 19, 1991, pp. 2468-2471. doi:10.1103/PhysRevLett.66.2468

[2] M. Botton and A. Ron, "Self-Induced Distributed Feedback in Plasma-Filled Cerenkov Free Electron Masers," Physical Fluids B, Vol. 4, No. 7, 1992, pp. 1979-1988. doi:10.1063/1.860051

[3] A. T. Lin and L. Chen, "Plasma-Induced Efficiency Enhancement in a Backward Wave Oscillator," Physical Review Letters, Vol. 63, No. 26, 1989, pp. 2808-2811. doi:10.1103/PhysRevLett.63.2808

[4] Y. Yan and S. Liu, "Dispersion Characteristics of Plasma Mode in Corrugated Plasma Waveguide," International Journal of Infrared and Millimeter Waves, Vol. 20, No. 10, 1999, pp. 1725-1730. doi:10.1023/A:1021745127565

[5] B. H. P. Broks, J. Dijk, H. M. J. Bastiaens, K. J. Boller, and J. J. A. M. V. Mullen, "Study of a Pulsed Capillary Discharge Waveguide with a Modulated Radius," Journal of Physical D: Applied Physics, Vol. 39, No. 11, 2006, pp. 2384-2390. doi:10.1088/0022-3727/39/11/012

[6] H. Kogelnik and C. V. Shank, "Coupled-Wave Theory of Distributed Feedback Lasers," Journal of Applied Physics, Vol. 43, No. 5, 1972, pp. 2327-2335. doi:10.1063/1.1661499

[7] Y. C. Huang and Y. Y. Lin, "Coupled-Wave Theory for Distributed-Feedback Optical Parametric Amplifiers and Oscillators," Journal of the Optical Society of American B, Vol. 21, No. 4, 2004, pp. 777-790. doi:10.1364/JOSAB.21.000777

[8] N. Kim, S. Han, H. Ryu, H. Ryu, H. Ko, J. Park, D. Lee, M. Jeon and K. Park, "Distributed Feedback Laser Diode Integrated with Distributed Bragg Reflector for Continuous-Wave Terahertz Generation," The International Journal of Optics, Vol. 20, No. 16, 2012, pp. 17496-17502.

[9] B. D. Layer, A. York, T. M. Antonsen, S. Varma, Y.-H. Chen, Y. Leng and H. M. Milchberg, "Ultrahigh-Intensity Optical Slow-Wave Structure," Physical Review Letters, Vol. 99, No. 3, 2007, pp. 035001-035004. doi:10.1103/PhysRevLett.99.035001

[10] J. P. Palastro, T. M. Antonsen, S. Morshed, A. G. York and H. M. Milchberg, "Pulse Propagation and Electron Acceleration in a Corrugated Plasma Channel," Physical Review E, Vol. 77, No. 3, 2008, pp. 036405-036418. doi:10.1103/PhysRevE.77.036405

[11] A. G. York, B. D. Layer, J. P. Palastro, T. M. Antonsen and H. M. Milchberg, "Ultrahigh-Intensity Optical SlowWave Structure for Direct Laser Electron Acceleration," Journal of the Optical Society of American B, Vol. 25, No. 7, 2008, pp. 137-146. doi:10.1364/JOSAB.25.00B137

[12] Y. R. Shen, "The Principles of Nonlinear Optics," John Wiley \& Sons, New York, 1984. 